\title{
Isolated ascending aorta endografting: Another brush for the aortic reconstruction artist
}

\author{
Nicholas D. Andersen, MD
}

\footnotetext{
From the Division of Cardiovascular and Thoracic Surgery, Department of Surgery, Duke University Medical Center, Durham, NC.

Disclosures: Author has nothing to disclose with regard to commercial support.

Received for publication March 2, 2016; accepted for publication March 4, 2016; available ahead of print March $25,2016$.

Address for reprints: Nicholas D. Andersen, MD, Division of Cardiovascular and Thoracic Surgery, Duke University Medical Center, Box 3443, Durham, NC 27710 (E-mail: Nicholas.andersen@ duke.edu).

J Thorac Cardiovasc Surg 2016;151:1615-6

$0022-5223 / \$ 36.00$

Copyright (C) 2016 by The American Association for Thoracic Surgery

http://dx.doi.org/10.1016/j.jtcvs.2016.03.006
}

The art and science of aortic reconstruction continues to progress, and now includes established or emerging endovascular repair options for nearly all segments of the aorta. However, the endovascular revolution has been slow to engage the proximal aorta due to its complex regional anatomy, hemodynamic environment, and somewhat inaccessible location. Endovascular arch procedures using branched or fenestrated endografts or open arch debranching have proven feasible, but remain technically challenging and anatomically treacherous due to a reliance on ascending aorta proximal landing zone (Ishimaru zone 0$)^{1}$ with the attendant risks of device migration, coronary or arch vessel occlusion, and retrograde dissection. ${ }^{2}$ However, a more restrained application of proximal aorta endografting that avoids the technical challenges of arch vessel preservation entails isolated pavement of the supracoronary ascending aorta, from the sinotubular junction to the innominate artery, for select high-risk patients with focal aortic pathology contained wholly within this segment. However, this short arc of aorta is poorly accessible by standard percutaneous routes and no devices are specially designed for the unique length, diameter, and curvature specifications of the ascending aorta. In addition, given the proximity to the aortic root, coronary, and innominate artery ostia, isolated endograft placement within the ascending aorta risks sudden cardiac decompensation or debilitating stroke with any technical misstep or millimeter of device migration, and the ominous threat of retrograde dissection with zone 0 endograft placement continues to persist. ${ }^{2}$ Nonetheless, case reports and small case series of isolated ascending aorta endografting in patients with poor open repair options have slowly emerged, including 2 reports published last year in The Journal from the University of Pennsylvania $(\mathrm{n}=6)^{3}$ and Cleveland Clinic $(\mathrm{n}=22) .{ }^{4}$ In the current issue of The Journal, Galli and colleagues ${ }^{5}$ provide another insightful report of 8 cases of isolated ascending aorta endografting to add to this scarce literature.

In the current study from Galli and colleagues, ${ }^{5} 8$ highrisk patients with penetrating aortic ulcer or ascending

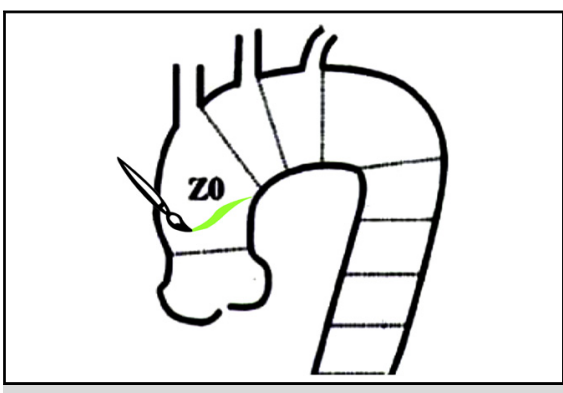

The art of aortic reconstruction continues to expand with isolated zone 0 endografting.

Central Message

Isolated ascending aorta endografting provides an intuitive repair solution for focal aortic pathology in select high-risk patients.

See Article page 1606.

aortic pseudoaneurysm deemed unsuitable for open repair were treated with isolated ascending aorta endografting at 2 centers in Italy. In contrast to the previously mentioned reports, ${ }^{3,4}$ patients with fusiform aneurysm or dissection were excluded, creating a more stable patient cohort without emergent operations. The technical details of device selection and delivery were generally similar to prior reports with the exception of 2 patients who underwent elective endografting with custom-manufactured devices (Relay; Bolton Medical, Sunrise, Fla) specifically designed to contour the precise specifications of their ascending aorta, including 1 patient who was treated with a custom-designed reverse-tapered device. In short, all 8 patients did well. There were no open conversions or intraoperative mishaps. One patient is being observed for an asymptomatic low-flow type 3 endoleak. Although the custom-manufactured devices were only used in 2 of 8 patients, the authors were clearly pleased with their performance over existing off-the-shelf technology and recommended their use "whenever possible."

Overall, these data demonstrate the excellent technical results and patient outcomes that can be achieved when focal aortic pathology is treated with isolated ascending aorta endografting in the nonemergent setting. Not surprisingly, these results differ somewhat from the report from Cleveland Clinic, where significant morbidity and technical challenges were encountered primarily when attempting these procedures in patients in extremis with acute dissection or aorta-cardiac fistula. ${ }^{4}$ Galli and colleagues ${ }^{5}$ should 


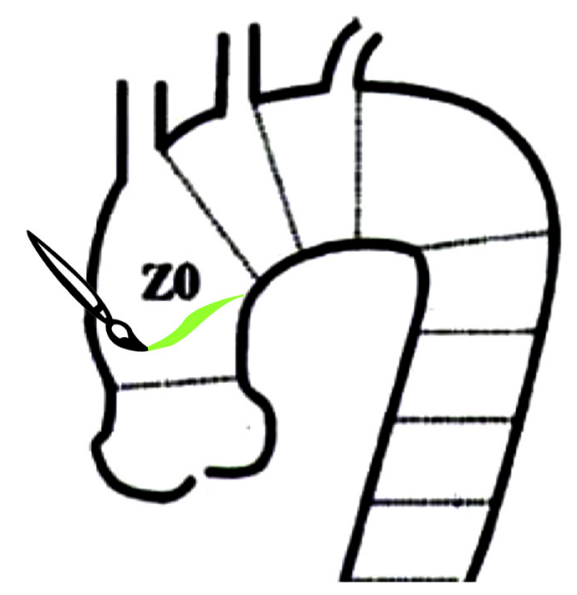

FIGURE 1. The art of aortic reconstruction continues to expand with isolated zone 0 endografting.

further be applauded for enrolling patients in surgery via an institutional review board-approved informed consent protocol after multidisciplinary team review, thereby acknowledging and disclosing to patients the unproven and experimental nature of these rare procedures at the outset. The only shortcoming of the report is that the finer details of patient selection, device selection, sizing, and custom graft design are slim. The precise exclusion criteria for open repair, the exact commercial devices selected and rationale for use in each scenario, and the algorithm for custom endograft design were not clearly specified. Nonetheless, the patient data and detailed aorta and endograft measurements provided from each case allow the reader to decipher the general decision-making strategy.

In conclusion, the study by Galli and colleagues ${ }^{5}$ expands the clinical evidence and technical knowledge for isolated ascending aorta endografting, representing yet another specialized technique in the repertoire of the aortic reconstruction artist (Figure 1). However, endograft placement in the ascending aorta is a technical feat not to be underestimated, in part due to the lack of tailored ascending aorta endografts and delivery systems, and the uncertain risks of retrograde dissection and device migration must also be acknowledged. Thus, as was prudently recognized by Galli and colleagues ${ }^{5}$ and others, ${ }^{3,4}$ these remain highly experimental "off-label" procedures that should be reserved for expert proximal aorta technicians and anatomically appropriate patients with limited open repair options.

\section{References}

1. Ishimaru S. Endografting of the aortic arch. J Endovasc Ther. 2004;11(Suppl 2): II62-71.

2. Benrashid E, Wang H, Keenan JE, Andersen ND, Meza JM, McCann RL, et al. Evolving practice pattern changes and outcomes in the era of hybrid aortic arch repair. J Vasc Surg. 2016;63:323-31.e1.

3. Vallabhajosyula P, Gottret JP, Bavaria JE, Desai ND, Szeto WY. Endovascular repair of the ascending aorta in patients at high risk for open repair. J Thorac Cardiovasc Surg. 2015;149(2 Suppl):S144-50.

4. Roselli EE, Idrees J, Greenberg RK, Johnston DR, Lytle BW. Endovascular stent grafting for ascending aorta repair in high-risk patients. J Thorac Cardiovasc Surg. 2015; 149:144-51.

5. Galli M, Piffaretti G, Lomazzi C, Franchin M, Castelli P, Mariscalco G, et al. Endograft repair for pseudoaneurysms and penetrating ulcers of the ascending aorta. J Thorac Cardiovasc Surg. 2016;151:1606-14. 